\title{
The Influence of Material and Mesh Characteristics on Transmission Mode Desorption Electrospray Ionization
}

\author{
Joseph E. Chipuk and Jennifer S. Brodbelt \\ Department of Chemistry and Biochemistry, University of Texas at Austin, Austin, Texas, USA
}

\begin{abstract}
Adaptation of desorption electrospray ionization to a transmission mode (TM-DESI) entails passing an electrospray plume through a sample that has been deposited onto a mesh substrate. A combination of mass spectrometry and fluorescence microscopy studies is used to illustrate the critical role material composition, mesh open space, and mesh fiber diameter play on the transmission, desorption, and ionization process. Substrates with open spaces less than $150 \mu \mathrm{m}$ and accompanying minimal strand diameters produce less scattering of the plume and therefore favor transmission. Larger strand diameters typically encompass larger open spaces, but the increase in the surface area of the strand increases plume scattering as well as solvent and analyte spreading on the mesh. Polypropylene (PP), ethylene tetrafluoroethylene (ETFE), and polyetheretherketone (PEEK) materials afford much better desorption than similarly sized polyethylene terephthalate (PETE) or nylon-6,6 (PA66) substrates. Ultimately, the manner in which the electrospray plume interacts with the mesh as it is transmitted through the substrate is shown to be critical to performing and optimizing TM-DESI analyses. In addition, evidence is presented for analyte dependent variations in the desorption mechanisms of dry and solvated samples. (J Am Soc Mass Spectrom 2009, 20, 584-592) () 2009 American Society for Mass Spectrometry
\end{abstract}

$\mathrm{D}$ esorption electrospray ionization (DESI) is among the growing number of atmospheric pressure ionization techniques that are suitable for coupling to mass spectrometric analysis [1]. In DESI, ions are produced by directing charged solvent droplets from an electrospray source toward a sample that is either a bulk material in its native state (e.g., pharmaceutical tablet) or one that has been deposited from solution onto a sampling surface. Analytes present at the surface are desorbed and ionized by the incoming plume and subsequently transferred to the mass spectrometer inlet by the influence of the applied potential and the pressure differential between atmospheric pressure and the low-pressure region of the mass analyzer. To date, DESI has found many applications including forensic analysis [2-5], explosives detection [6-9], chemical warfare agent detection [3, 10-12], imaging [13-15], pharmaceutical analysis [16-24], natural product characterization $[2,25]$, polymer analysis [26, 27], metabolomics [2, 28-31], proteomics [2, 32-33], and glycomics [34].

Recent adaptations of DESI, including geometry independent DESI in gas tight enclosures [35] and transmission mode desorption electrospray ionization (TM-DESI), have been developed to reduce the

Address reprints to: Jennifer S. Brodbelt, Department of Chemistry and Biochemistry, 1 University Station A5300, University of Texas at Austin, Austin, TX 78712; (512)-471-0028. E-mail: jbrodbelt@mail.utexas.edu geometry dependence of DESI experiments [36]. In the transmission DESI mode the sample is not deposited onto a continuous solid surface but rather onto a sampling mesh. In this adaptation, the incident spray angle and collection angle are fixed at $0^{\circ}$ and the spray is transmitted through the sample (Figure 1). Along with the simplification of the experimental geometry, the transmission mode also allows convenient analysis of both dry (i.e., following evaporation of the deposition solvent) and wet (i.e., solvated) samples with similar performance characteristics to those achieved using traditional DESI [36].

Surface variables including the chemical composition, porosity, texture, and electrical conductivity of the substrate have been reported to affect DESI analyses [2, 24, 37-39]. Dramatic reductions in response have been noted for high conductivity surfaces due to neutralization of the incoming ion plume at the surface $[2,38]$. Variations in response due to the impact of the surface on crystallization of deposited samples have been reported [2,37], as well as increases in response for porous or rough surfaces due to a reduction in sample spreading [2,37], and increases in response due to chemical inertness and hydrophobicity of materials such as PTFE [24, 38]. DESI analyses have utilized a variety of surface materials, including glass, PMMA, PTFE, TLC plates, UTLC plates, porous silicon, nanoporous aluminum, paper, and stainless steel. However, 


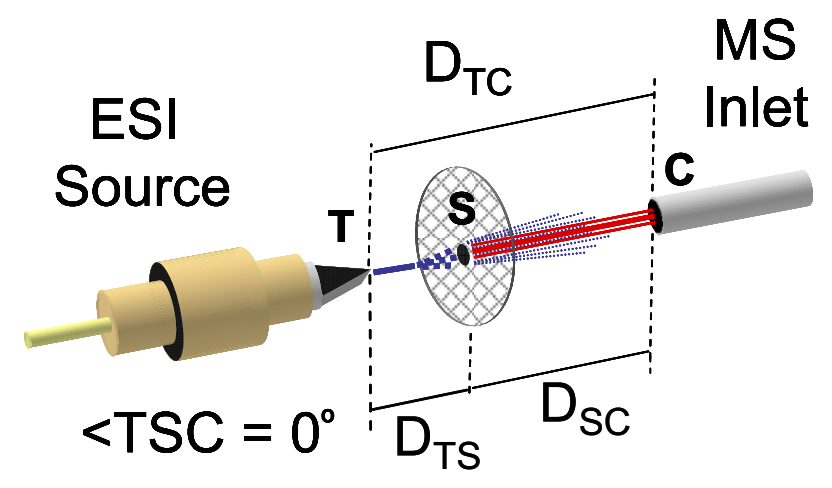

Figure 1. Transmission mode desorption electrospray ionization (TM-DESI) geometry. Sample (S) is deposited on a mesh screen and analyzed by passing an electrospray through it. The angle between the electrospray tip (T), sample (S), and capillary inlet (C) to the mass spectrometer is set to 0 degrees. $\mathrm{D}_{\mathrm{TC}}$ is the distance from the electrospray tip to the capillary, $\mathrm{D}_{\text {TS }}$ is the distance from the electrospray tip to the sample, and $\mathrm{D}_{\mathrm{SC}}$ is the distance from the sample to the capillary.

in general the use of rough, nonconducting materials is now widely preferred.

The utilization of a mesh material as the sample substrate for TM-DESI analysis introduces a new set of experimental variables that have not been investigated by traditional DESI. In TM-DESI, the mesh substrate is not only composed of a particular material, but also fashioned into a variety of different forms depending on the strand size used for the mesh and the open space between the various strands. Liquid samples spotted onto the sampling mesh fill one or more of the openings and the analyte may either adsorb onto the mesh or remain partitioned in the deposition solvent. Initial studies of rhodamine 6G, bradykinin, and nicotine by TM-DESI using mesh substrates composed of five dif- ferent materials but with similar mesh characteristics showed tremendous differences in response [36]. The present investigation expands on the previous results by including not only more materials but also substrates with widely varying mesh characteristics. Furthermore, more attention is given to the interaction of the electrospray plume with the mesh substrate, how the mesh characteristics impact the transmission of the electrospray, and how the material composition and mesh characteristics ultimately impact desorption and ionization mechanisms of liquid samples by TM-DESI.

\section{Experimental}

\section{Materials}

Rhodamine 6G and bradykinin were purchased from Sigma Aldrich (St. Louis, MO) and used without further purification. High purity methanol, water, and acetone were purchased from Fisher Scientific (Hampton, NH). Working standards of both rhodamine 6G and bradykinin were prepared at concentrations of 60 and 100 $\mathrm{pg} / \mu \mathrm{L}$ in methanol.

A total of 20 different mesh substrates manufactured from five different polymeric materials; polypropylene (PP), polyetheretherketone (PEEK), ethylene tetrafluoroethylene (ETFE), nylon-6,6 (PA66), and polyethylene terephthalate (PETE) were purchased from Small Parts, Inc. (Miramar, FL). The monomeric structures for these materials are shown in Figure 2. As indicated in Table 1, the different materials were fashioned into meshes with differing structural characteristics, namely the open space between the strands of the mesh and the diameter of the strands used to compose the mesh. Ultimately, these two variables were used to compute a theoretical percent transmittance of the mesh by taking the square<smiles>CCC(C)C</smiles>

polypropylene (PP)

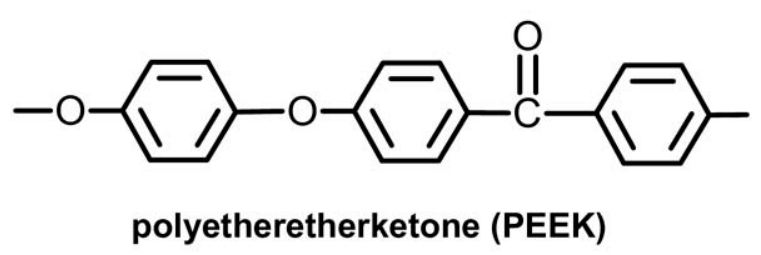

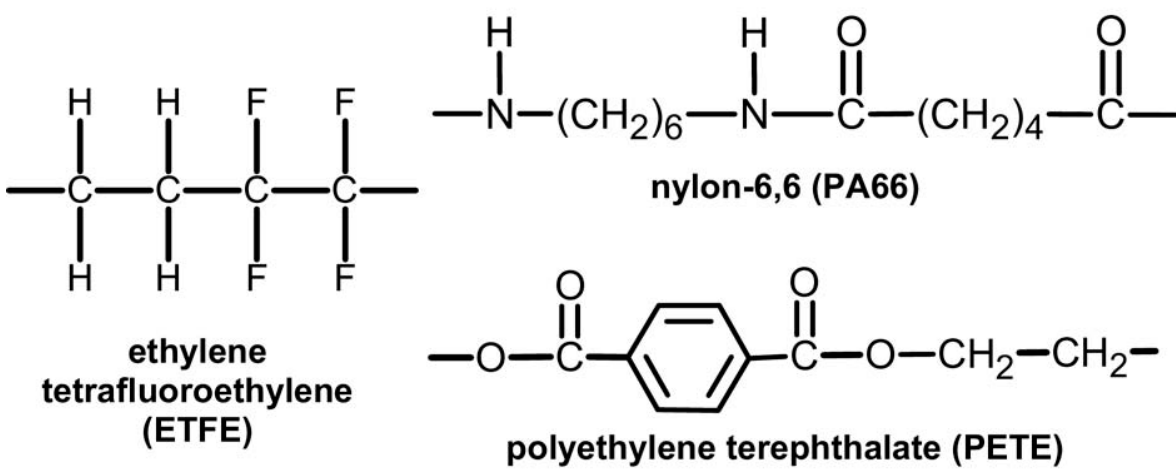

Figure 2. Monomers of the polymeric mesh materials used as TM-DESI substrates. 
Table 1. Sample mesh characteristics and results summary for rhodamine $6 \mathrm{G}$ analyses

\begin{tabular}{|c|c|c|c|c|c|c|}
\hline $\begin{array}{c}\text { Mesh } \\
\text { material }\end{array}$ & $\begin{array}{c}\text { Open space } \\
(\mu \mathrm{m})\end{array}$ & $\begin{array}{c}\text { Strand } \\
\text { diameter }(\mu \mathrm{m})\end{array}$ & $\begin{array}{c}\text { Transmittance } \\
(\%)^{\mathrm{a}}\end{array}$ & $\begin{array}{c}\text { Average } \\
\text { recovery }(\%)^{\mathrm{b}}\end{array}$ & $\begin{array}{c}\text { Normalized } \\
\text { recovery }(\%)^{\mathrm{c}}\end{array}$ & $\begin{array}{c}\text { Normalized } \\
\text { desorption }(\%)^{d}\end{array}$ \\
\hline PP & 105 & 100 & 26.2 & 33.5 & 68.6 & 56.1 \\
\hline PP & 125 & 100 & 30.9 & 40.3 & 82.6 & 55.4 \\
\hline PP & 149 & 106 & 34.1 & 48.8 & 100.0 & 100.0 \\
\hline PP & 250 & 200 & 30.9 & 27.9 & 57.2 & 10.1 \\
\hline PP & 297 & 215 & 33.6 & 37.7 & 77.2 & 6.3 \\
\hline PP & 500 & 300 & 39.1 & 23.0 & 47.2 & 4.3 \\
\hline PA66 & 110 & 51 & 46.7 & 15.6 & 31.9 & 27.6 \\
\hline PA66 & 130 & 50 & 52.2 & 22.5 & 46.1 & 19.7 \\
\hline PA66 & 150 & 92 & 38.4 & 7.1 & 14.5 & 15.9 \\
\hline PA66 & 250 & 180 & 33.8 & 3.7 & 7.6 & 3.7 \\
\hline PA66 & 310 & 151 & 45.2 & 8.0 & 16.3 & 7.6 \\
\hline PA66 & 500 & 315 & 37.6 & 2.0 & 4.1 & 14.2 \\
\hline PETE & 105 & 42 & 51.0 & 10.8 & 22.2 & 5.8 \\
\hline PETE & 132 & 54 & 50.4 & 5.1 & 10.5 & 6.8 \\
\hline PETE & 150 & 96 & 37.2 & 4.5 & 9.2 & 2.0 \\
\hline PETE & 250 & 152 & 38.7 & 12.0 & 24.6 & 18.8 \\
\hline PETE & 300 & 143 & 45.9 & 10.0 & 20.5 & 19.9 \\
\hline PETE & 500 & 220 & 48.2 & 13.6 & 27.9 & 10.2 \\
\hline ETFE & 150 & 96 & 37.2 & 40.6 & 83.2 & 86.9 \\
\hline PEEK & 300 & 200 & 36.0 & 24.5 & 50.3 & 21.5 \\
\hline
\end{tabular}

aThe percent transmittance was calculated as the square of the open space divided by the square of the sum of the open space and strand diameter. bThe average recovery was calculated as the average response of ten replicate electrospray analyses when the mesh was placed in the path of the electrospray plume divided by the response of the same solution electrosprayed without any mesh present in the sample holder. All values are $\pm 0.5 \%$.

${ }^{\mathrm{c}}$ The average response of ten replicate electrospray transmission analyses relative to all of the mesh materials. All values are $\pm 0.5 \%$.

${ }^{\mathrm{d}}$ The average response of ten replicate TM-DESI analyses relative to all of the mesh materials. All values are $\pm 0.5 \%$.

of the open space divided by the square of the sum of the open space and strand diameter. Throughout the text, mesh materials are given an alphanumeric designation by their material composition and their open space. (e.g., PP 149 was the mesh composed of polypropylene fibers with an open space of $149 \mu \mathrm{m}$ ) Before analysis, each mesh substrate was rinsed with a mixture of high purity water, methanol, and acetone. After drying, the mesh sheets were cut into $5 \mathrm{~mm} \times 10 \mathrm{~mm}$ rectangular pieces. Blank measurements were made on representative samples of each substrate to ensure that each was free of analyte or any detectable interference.

\section{Mass Spectrometry}

An Omni Spray ion source (Prosolia, Inc., Indianapolis, IN) was mounted to a Thermo Fisher Scientific LTQ XL mass spectrometer (Thermo Fisher Scientific Inc., Waltham, MA) and modified to allow a $0^{\circ}$ angle between the electrospray tip and capillary inlet to the mass spectrometer. Samples were affixed to the sample slide arm of the Omni Spray ion source using a sample holder constructed of two rectangular support pieces $(1.2 \mathrm{~cm} \times 15 \mathrm{~cm})$ : one high density polyethylene (HDPE) $2.3 \mathrm{~mm}$ thick and one oriented polyester 0.3 $\mathrm{mm}$ thick. A $7 \mathrm{~mm}$ diameter hole was drilled through both support pieces and the sample mesh was held between the two layers. Mass spectra were acquired by scrolling the sample holder perpendicularly into the electrospray plume between the spray tip and the cap- illary inlet, thereby allowing transmission of the ionizing spray through the mesh. Except where otherwise noted, all analyses were carried out with a TMDESI geometry of $\mathrm{D}_{\text {TS }}$ equal to $2 \mathrm{~mm}$ and $\mathrm{D}_{\text {TC }}$ equal to $8 \mathrm{~mm}$ (see Figure 1).

Mass spectra were acquired using the Xcalibur 2.0 software program in the positive ion mode with the electrospray voltage set to $4.0 \mathrm{kV}$, the ion accumulation time set to $10 \mathrm{~ms}$ and signal averaging set for four microscans. Nitrogen at a pressure of 100 psi was used as the nebulizing gas. Unless otherwise noted, a syringe pump (Harvard Apparatus, Holliston, MA) was used to deliver either methanolic samples for ESI or methanol electrospray solvent for TM-DESI at a rate of $5 \mu \mathrm{L} / \mathrm{min}$. TM-DESI samples were prepared by spotting $1 \mu \mathrm{L}$ of solution onto a sample mesh using a $5 \mu \mathrm{L}$ syringe (SGE, Austin, TX) and analyzed wet, before the deposition solvent had evaporated.

The response of rhodamine 6G was monitored using a selected dissociation reaction of the protonated species of $\mathrm{m} / \mathrm{z} 443.3 \mathrm{Da}$ to the dominant fragment of $\mathrm{m} / \mathrm{z}$ 415.2 Da (i.e., selected reaction monitoring). The response of bradykinin was monitored using a selective ion monitoring of the doubly protonated species $[\mathrm{M}+$ $2 \mathrm{H}]^{2+}$ of $\mathrm{m} / \mathrm{z} 530.7 \mathrm{Da}$. The area of the peak chosen by Xcalibur's ICIS peak detection algorithm was used to compare responses and each experiment was repeated a minimum of five times to ensure consistency between mesh samples. 


\section{Fluorescence Microscopy}

Analysis of residual rhodamine 6G was performed using either the 2.5X or $4 \mathrm{X}$ objective of an Olympus BX2 epifluorescent microscope equipped with a 12 bit CCD camera (DVC Co., Austin, TX) and high-pressure mercury bulb excitation source. Excitation of rhodamine 6G occurred at $480 \mathrm{~nm}$ and emission was monitored at 535 $\mathrm{nm}$. Photomicrographs were captured via DVC software with adjustable gain, offset, and exposure time. While various microscopy settings were used for different experiments based on the sensitivity of the instrument and the amount of residual analyte, all microscopy settings were consistent among the samples being compared.

\section{Results and Discussion}

Desorption electrospray ionization involves the interaction of an incoming electrospray plume with analytes adsorbed onto a surface. Desorption and ionization of these analytes is believed to proceed through a multistep droplet pick-up mechanism, where the initial step involves the formation of a thin solvent layer on the surface by the incoming solvent plume [1, 40]. Adsorbed molecules eventually dissolve or migrate into the solvent layer and are subsequently desorbed by the momentum of the high velocity electrospray droplets that continue to contact the surface. Once the analytes are sequestered in the charged off-spring droplets, they are ionized by typical ESI mechanisms. Ultimately, the droplet pick-up mechanism depends not only on the characteristics of the electrospray but also on the physical and chemical characteristics of the surface. Specifically, the surface influences the formation of the solvent layer, spreading of the solvent layer, the ease with which off-spring droplets leave the surface and the migration rate of adsorbed molecules into the solvent layer. Therefore, experiments were designed to assess the size and shape of the electrospray plume interacting with the mesh, the impact of the mesh characteristics on electrospray transmission, and the influence of the mesh on the desorption of solvated analytes.

\section{Sample Spot Size and Shape in TM-DESI}

Experiments were conducted to determine the influence of the electrospray solvent flow rate and experimental geometry on the TM-DESI sampling spot size and shape. Accordingly, a standard piece of printer paper was cut into $5 \mathrm{~mm} \times 10 \mathrm{~mm}$ pieces and inserted into the sample holder in place of a sampling mesh. A solution of rhodamine $6 \mathrm{G}(100 \mathrm{pg} / \mu \mathrm{L}$ in $\mathrm{MeOH})$ was electrosprayed for $30 \mathrm{~s}$ at various flow rates $(2 \mu \mathrm{L} / \mathrm{min}$ to 10 $\mu \mathrm{L} / \mathrm{min}$ ) and distances $\left(\mathrm{D}_{\mathrm{TS}} 2 \mathrm{~mm}\right.$ to $8 \mathrm{~mm}$ ) onto the paper substrate, resulting in depositions of 100 to 500 pg of rhodamine 6G.

Fluorescence microscopy was used to determine the effective size and shape of the electrospray plume as it reached the substrate. Figure 3 shows an example

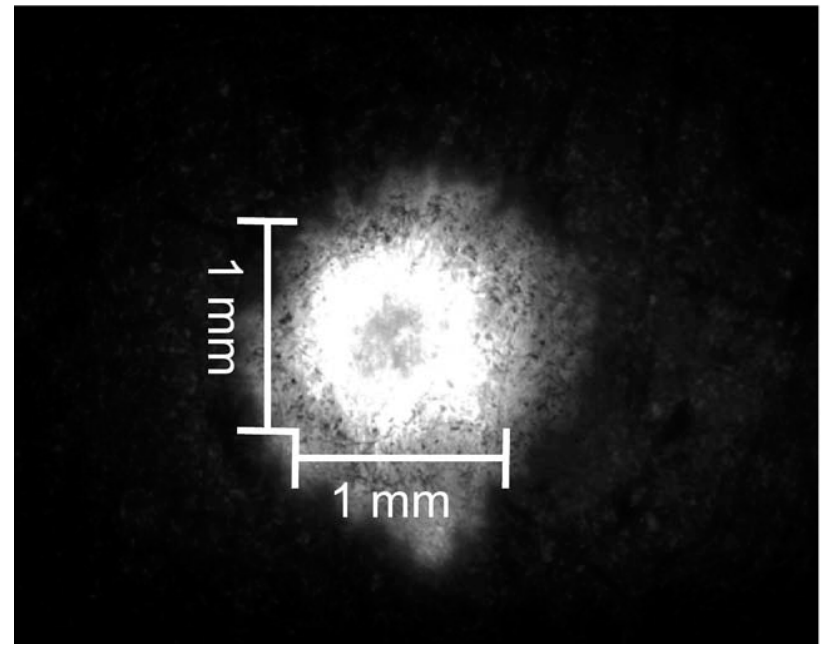

Figure 3. Fluorescence image of rhodamine $6 \mathrm{G}$ electrosprayed onto a paper substrate at a distance of $2 \mathrm{~mm}$ and an electrospray flow rate of $5 \mathrm{uL} / \mathrm{min}$. Under these electrospray conditions the TM-DESI spot size remains essentially circular and has a diameter of $\sim 1 \mathrm{~mm}$.

where the electrospray flow rate was $5 \mu \mathrm{L} / \mathrm{min}$ and the distance from the spray tip to the sample was $2 \mathrm{~mm}$. The bright area shown in the photomicrograph illustrates that the TM-DESI spot is dense, relatively symmetrical, and has a diameter of $\sim 1 \mathrm{~mm}$ under these electrospray conditions. These characteristics therefore result in an effective sampling area of $\sim 0.8 \mathrm{~mm}^{2}$. Higher flow rates and larger distances resulted in more irregular, diffuse spray patterns with sampling diameters about 2 to $3 \mathrm{~mm}$. These results generally agree with recent reports for DESI imaging [15] but differ in that elliptical spray patterns are not observed at higher flow rates. Ultimately, a distance of $2 \mathrm{~mm}$ and an electrospray flow rate of $5 \mu \mathrm{L} /$ min were chosen for all subsequent analyses as a balance between spot regularity and the flux of the ionizing solvent through the mesh.

\section{Electrospray Transmission through Mesh Substrates}

Numerous studies have investigated fundamental electrospray characteristics at various geometries, flow rates, gas pressures and applied voltages [41-43]. However, the transmission of an electrospray through a material is not typically addressed. One way to probe the interaction is to compare the mass spectral response when an analyte is electrosprayed through the mesh with that when no mesh is present. Accordingly, a solution of rhodamine $6 \mathrm{G}(60 \mathrm{pg} / \mu \mathrm{L}$ in $\mathrm{MeOH})$ was electrosprayed at a flow of $5 \mu \mathrm{L} / \mathrm{min}$ though each mesh material for $20 \mathrm{~s}$, resulting in the analysis of a total of $100 \mathrm{pg}$ of rhodamine 6G. Ten replicate experiments were conducted for each mesh material, each utilizing a new mesh for the analysis. The average response when 
the mesh was present was divided by the average response obtained in control experiments (i.e., no mesh present) performed immediately before and after each set of replicates, thereby resulting in an average percent recovery for rhodamine 6G. These results, along with the normalized percent recovery among the twenty different mesh substrates, are presented in Table 1.

The average recovery of rhodamine $6 \mathrm{G}$ varied from $2.0 \%$ to $48.8 \%$, clearly indicating that the physical parameters of the mesh substrate had a dramatic impact on the transmission of the analyte. Examination of the entire dataset illustrates that the transmission of rhodamine 6G is not strictly favored for mesh materials with large open spaces, as materials such as PP 149 and ETFE 150 yield higher recoveries than PA66 500 or PETE 300, even though the open space is one-half to one-third as large. Furthermore, high recoveries were not strictly attributed to small strand diameters, as recoveries for PP 297 and PA66 500 are much larger than ETFE 105 and PA66 150, although the latter have much smaller strand diameters. When substrates are grouped based on their open space (e.g., PP 149, ETFE 150, PA66 150, PETE 150), it is apparent that PP, ETFE, and PEEK materials afford much higher transmission than similarly sized PETE or PA66 substrates, especially for those with open spaces less than $300 \mu \mathrm{m}$ (see Figure 4). These results are consistent throughout each size grouping and emphasize the influence of the mesh material relative to the size characteristics of the mesh. However, the size characteristics are also an influential factor since the average recoveries for each mesh of a particular material were not equal.

Rhodamine $6 \mathrm{G}$ that was not transmitted through the mesh to the mass spectrometer must have either adsorbed to the mesh or been scattered by the mesh away from the capillary inlet. Therefore, the mesh samples used for the transmission experiments were analyzed by fluorescence microscopy to determine the extent of analyte adsorption that occurred during the analysis. Furthermore, the transmission experiments were also repeated with a second sample holder containing a 5 $\mathrm{mm} \times 10 \mathrm{~mm}$ piece of paper placed $\sim 6 \mathrm{~mm}$ behind the sample holder containing the mesh material but directly in front of the capillary inlet. In this case, the paper was used to collect rhodamine 6G analyte (i.e., ions and solvated neutrals) that was scattered by the various mesh substrates.

As shown in Figure 5, there was a remarkable difference in the photomicrographs of the mesh materials. Materials such as PETE and PA66 tended to adsorb the rhodamine $6 \mathrm{G}$ while those composed of PP or ETFE did not. These results correlate directly with the recovery data and may be explained by either the porosity of the strands or the polarity and charging characteristics of the oligomeric materials since hydrophobic materials such as PP did not adsorb the dye nearly as much as the PA66 or PETE. Furthermore, the variation among the series of substrates of a particular material was at least partially explained by the amount of visible spreading of the adsorbed analyte. While the mesh materials with small open spaces and small strand diameters showed little or no adsorption of analyte outside of the $\sim 1 \mathrm{~mm}$ spot size, the meshes with large open spaces and correspondingly larger strand sizes were typically observed to have rhodamine $6 \mathrm{G}$ spread several $\mathrm{mm}$ across the mesh. These results suggest that the erosion effects typical in DESI [44] may also be present in TM-DESI, but only when the surface area of the mesh strands becomes too large for effective transmission.

The results of the scattering experiments also demonstrated that mesh materials with larger strand sizes (i.e., those with open spaces $\sim 150$ to $315 \mu \mathrm{m}$ ) tended to facilitate scattering of the analytes as they passed through the mesh. For example; PP 250, PP 297, PETE 300, and PA66 310 all showed the distinct deposition of rhodamine $6 \mathrm{G}$ in multiple locations on the paper surrounding the capillary inlet. In contrast, transmission of the electrospray through mesh materials with smaller stand diameters (i.e., $\sim 100 \mu \mathrm{m}$ ), such as PETE 105, PETE 50, ETFE 150, and PP 105, did not appear to produce any significant scattering (see Supplementary Figure S1, which can be found in the electronic version of this article).
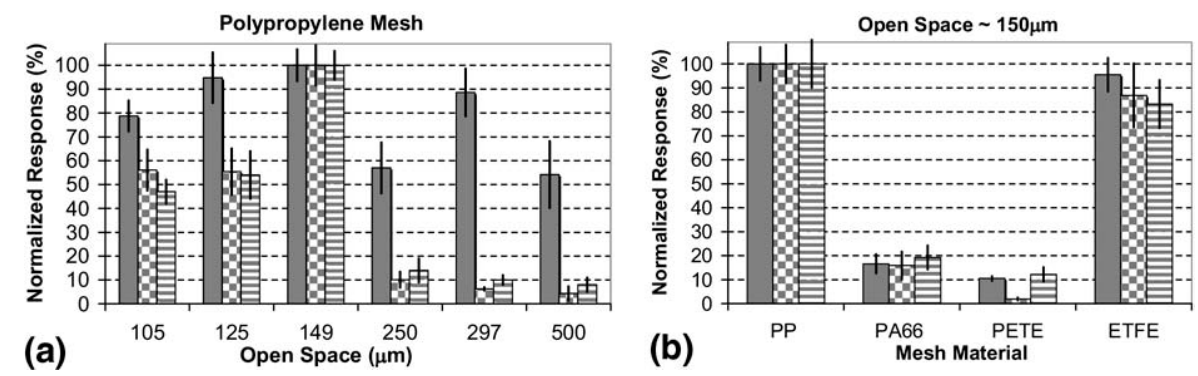

Figure 4. Normalized responses of rhodamine 6G and bradykinin using (a) polypropylene mesh materials with open spaces ranging from 105 to $500 \mu \mathrm{m}$ and (b) meshes of different materials with open spaces of $\sim 150 \mu \mathrm{m}$. Solid bars indicate transmission of rhodamine $6 \mathrm{G}$ electrosprayed through the mesh. Square patterned bars correspond to desorption of rhodamine $6 \mathrm{G}$ while horizontal striped bars correspond to desorption of bradykinin using TM-DESI analysis. 

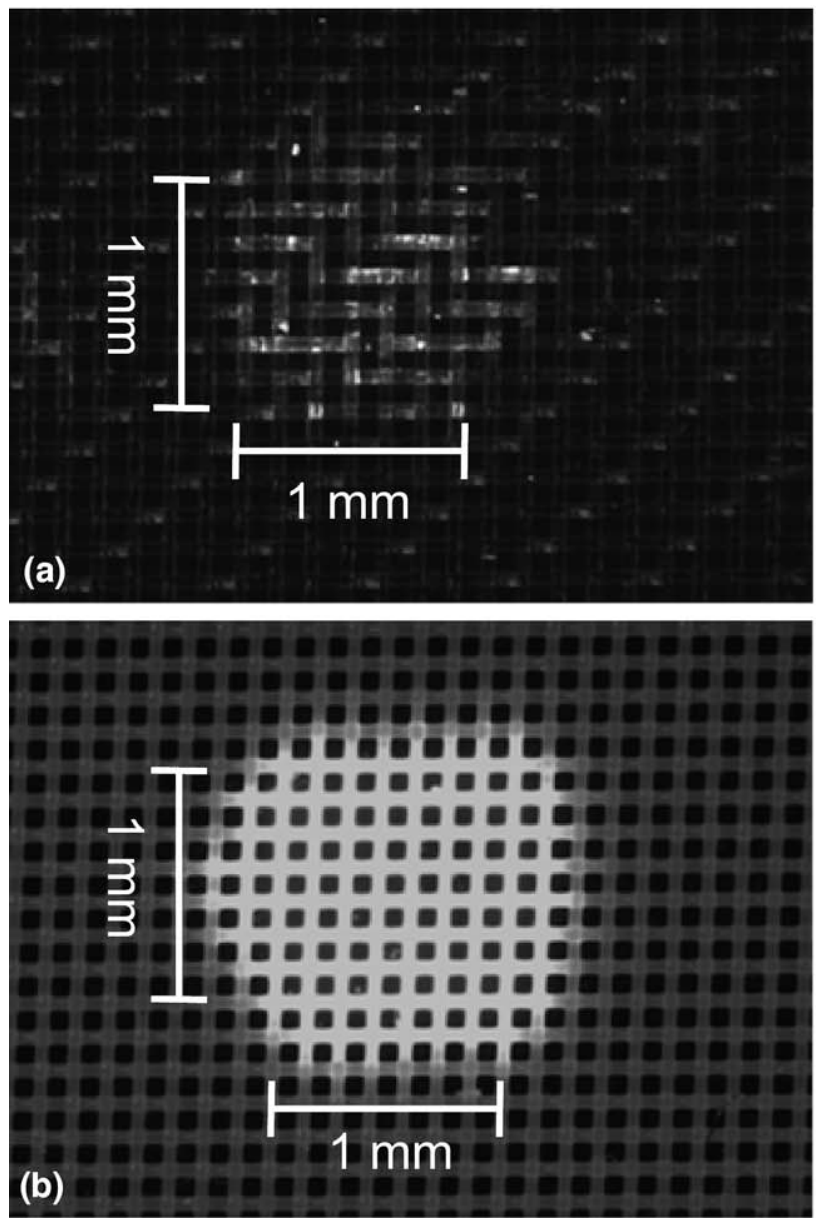

Figure 5. Fluorescence micrographs of mesh materials (a) PP 149 (149 $\mu \mathrm{m}$ open space) and (b) PETE $150(150 \mu \mathrm{m}$ open space) following electrospray of rhodamine $6 \mathrm{G}(60 \mathrm{pg} / \mu \mathrm{L})$ through the mesh for $20 \mathrm{~s}$. Both micrographs were acquired using identical microscope gain and exposure settings.

\section{Desorption of Liquid Samples from Mesh Substrates}

Experiments were also performed to investigate the potential differences between desorption and transmission and their relationships to TM-DESI analysis of wet samples. Successful sample deposition for conventional DESI analyses involves balancing drying time and sample spreading. If low volatility solvents (e.g., water) are used, the drying time often becomes the rate limiting step of the analyses $[2,15]$. If a more volatile solvent is used (e.g., methanol), the evaporation time may be reduced; however the low surface tension of the solvent makes uniform sample deposition more challenging [2]. Furthermore, sample analyses that utilize smooth surfaces are typically prone to erosion effects, thereby requiring alternative deposition onto porous or rough surfaces that retain the adsorbed analyte during the formation of the initial solvation layer $[2,37,39]$. The mesh materials used for TM-DESI overcome these difficulties by suspending both high and low volatility deposition solvents of varying surface tensions in the open space of a mesh. Like DESI, TM-DESI is also expected to proceed through a droplet pickup mechanism. However, a distinction can be drawn between TM-DESI analyses of dry samples versus wet samples. Analysis of dry samples should be completely analogous to traditional DESI as analytes are merely adsorbed onto a mesh strand instead of a hydrophobic spot or other surface. However, wet analyses present a different situation since the deposition solvent remains a component of the system and effectively acts as an abundant initial solvent layer.

Analyte molecules that remain suspended in the droplets are efficiently and rapidly desorbed into the electrospray, where surface active analytes may be ionized by heterogeneous charge-transfer between the electrospray droplets and the analyte or by typical ESI mechanisms following droplet fusion. Both of these mechanisms have been proposed previously for electrospray assisted desorption techniques such as ELDI and MALDESI [45-50]. In contrast, analyte molecules that preferentially adsorb to the mesh substrate withstand the release of the deposition solvent droplets and instead are both desorbed and ionized by the spray solvent. The intricacies of the three component system (i.e., substrate, deposition solvent, electrospray solvent) are not entirely inconsequential as analyte response has been observed to depend on the identity of both the deposition and electrospray solvent [36].

Experiments analogous to the transmission studies were performed to assess the influence of various mesh materials and mesh characteristics on the desorption and ionization process. In this case, methanol was used as both the deposition and electrospray solvents, thereby isolating the experiment from any solventdependent mechanistic variations. Samples were prepared by depositing $1 \mu \mathrm{L}$ of a solution of rhodamine $6 \mathrm{G}$ $(100 \mathrm{pg} / \mu \mathrm{L})$ or bradykinin $(100 \mathrm{pg} / \mu \mathrm{L})$ on the surface of a mesh substrate and analyzed before solvent evaporation by scanning a single pass across the visibly wet surface at a rate of $300 \mu \mathrm{m} / \mathrm{s}$. Ten replicate experiments were conducted for each mesh material, each utilizing a new $5 \mathrm{~mm} \times 10 \mathrm{~mm}$ mesh. The response of the 10 measurements was averaged and normalized to the maximum average response for the 20 mesh substrates. The normalized results for rhodamine 6G desorption (i.e., normalized desorption) are presented alongside the transmission results (i.e., normalized recovery) in Table 1.

In general, the desorption of rhodamine $6 \mathrm{G}$ and bradykinin followed the trends established by the transmission experiments as the largest responses were observed for nonpolar mesh materials with moderate open space and small strand diameters. For both analytes, desorption from PP 149 and ETFE 150 resulted in the largest responses while much smaller signals were observed for substrates of different materials with similar mesh characteristics (e.g., PA66 150, PETE 150). Interestingly, results for other relatively transmissive substrates such as PP 250, PP 297, PP 500, and PK 300 
showed dramatic decreases in the relative efficiencies of desorption compared with transmission. Figure 4a highlights the differences between the various polypropylene substrates and Figure $4 \mathrm{~b}$ compares the responses for substrates with $\sim 150 \mu \mathrm{m}$ of open space. While the differences among the various mesh materials are of specific interest to this study, the relative similarity of the normalized responses for rhodamine $6 \mathrm{G}$ and bradykinin desorption by TM-DESI are particularly significant because they indicate the potential transference between a model compound such as rhodamine 6G and broader molecular classes such as peptides.

Fluorescence microscopy was again used to assess the impact of the mesh material by examining the extent of residual rhodamine $6 \mathrm{G}$ on the mesh after TM-DESI analysis. For example, Table 1 and Figure 4 indicate that the response of rhodamine 6G from PA66 via desorption was only $16 \%$ of the response from PP 149 . Figure 6 depicts example micrographs taken on PP 149 and
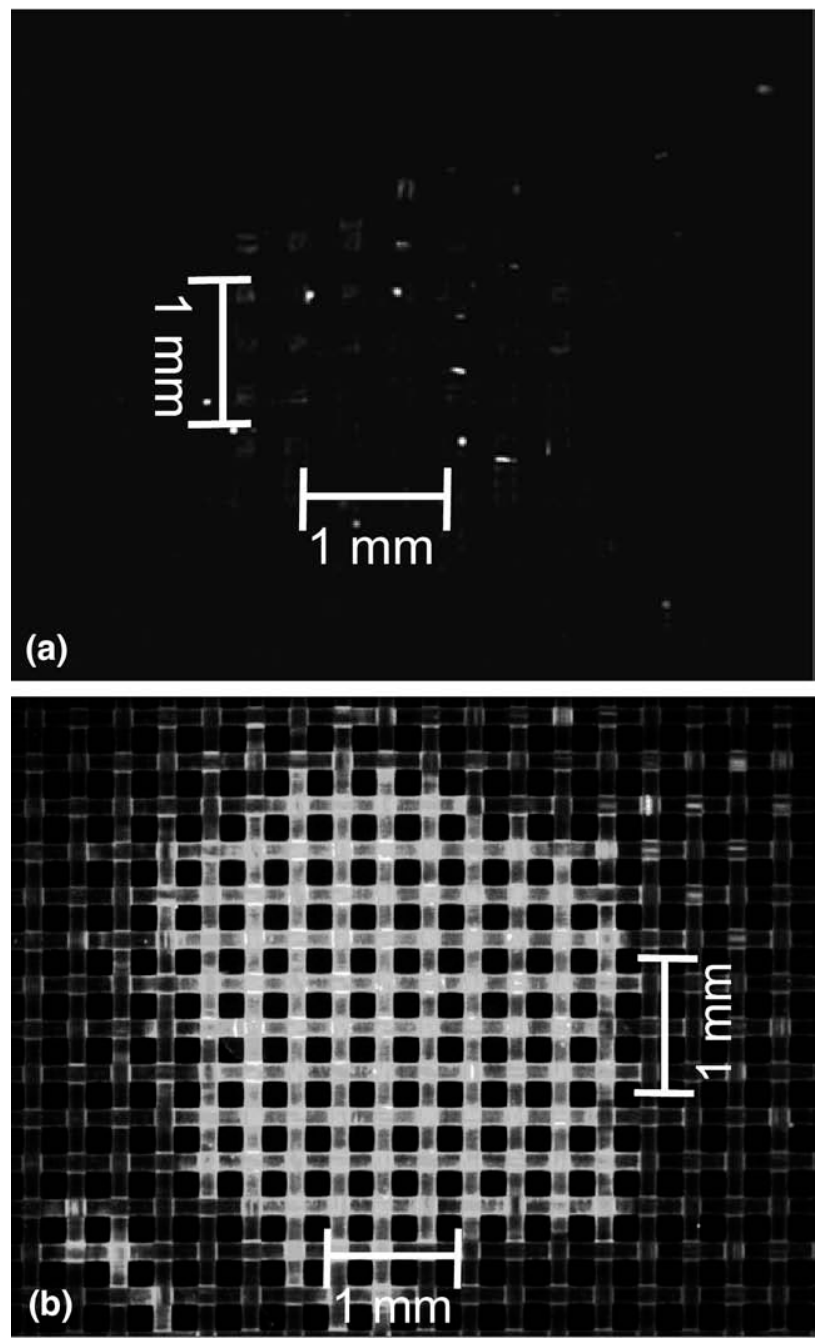

Figure 6. Fluorescence micrographs of mesh materials (a) PP 149 (149 $\mu \mathrm{m}$ open space) and (b) PA66 150 (150 $\mu \mathrm{m}$ open space) following TM-DESI analysis of a $1 \mu \mathrm{L}$ sample of rhodamine $6 \mathrm{G}$ (100 pg/ $\mathrm{LL}$ ). Both samples were analyzed wet and micrographs were taken using identical gain and exposure settings.
PA66 150 following these analyses. The differences clearly illustrate that the lower recovery is due to the preferential partitioning of the analyte between the substrate and the deposition solvent. In the case of PP 149 , the rhodamine $6 \mathrm{G}$ remained dissolved in the methanol and was thus rapidly desorbed and ionized by the electrospray plume as it was scanned across the mesh. In contrast, the PA66 mesh material more strongly bound the analyte and less of the dye remained in the deposition solvent. In this instance, scanning across the mesh removed the deposition solvent but left a significant amount of the analyte behind.

Additional experiments to assess the decay rate of rhodamine 6G spotted onto PP 149 and PA66 150 were performed to expand on these observations. In this case the electrospray was not scanned across the $1 \mu \mathrm{L}$ spot but instead moved rapidly to the position of the liquid droplet and held in place. The results for PP 149 echoed those previously reported for meshes of this material but of different size characteristics [36] and showed a sharp initial response for PP 149 followed by rapid signal decay. In contrast, the initial response from PA66 150 was much smaller and decayed more slowly, thereby indicating that the rhodamine $6 \mathrm{G}$ was gradually being desorbed from the mesh long after the initial deposition solvent had been incorporated into the electrospray plume.

\section{Conclusions}

The mass spectrometry and fluorescence microscopy results reported here illustrate the significance of the material of composition and the mesh characteristics on TM-DESI analyses. Under typical conditions, the electrospray plume will produce a relatively symmetrical circular sampling spot of $\sim 1 \mathrm{~mm}$ diameter. However, the transmission of the electrospray plume through the mesh depends highly on mesh characteristics such as open space and strand diameter. Substrates with open spaces less than $150 \mu \mathrm{m}$ and accompanying minimal strand diameters produce less scattering of the plume and therefore favor transmission. Larger strand diameters typically encompass larger open spaces, but the increase in the surface area of the strand increases both plume scattering and solvent and analyte spreading on the mesh.

Desorption of liquid samples from various mesh substrates is highly dependent on the material composition of the mesh and the relative affinity of the target analytes for the deposition solvent relative to the substrate material. Analytes that remain partitioned into the deposition solvent are rapidly desorbed into the electrospray plume where they undergo ionization via heterogeneous charge-transfer with other electrospray solvent droplets or by droplet fusion, charge redistribution and subsequent fission of the new progeny droplets. In contrast, analytes that have a higher affinity for the mesh substrate are desorbed more gradually from the surface following resolvation by the electrospray 
solvent in a typical droplet pickup mechanism. Experiments utilizing rhodamine $6 \mathrm{G}$ and bradykinin, typical model compounds for DESI analyses, clearly illustrate these effects as mesh materials composed of polypropylene and ethylene tetrafluoroethylene with open spaces of $\sim 150 \mathrm{um}$ and strand diameters of $\sim 100 \mu \mathrm{m}$ afforded the most efficient desorption, and ultimately the greatest response for both analytes.

The compilation of these results highlights the intricate chemistry that exists as the electrospray plume is transmitted through a mesh material, especially one containing a solvated sample. Ongoing research aims to take advantage of these studies by developing solventsurface systems that increase the selectivity of the transmission mode approach to desorption electrospray ionization.

\section{Acknowledgments}

The authors gratefully acknowledge funding from the Welch Foundation (F1155) and the National Science Foundation (CHE0718320). Additional recognition is also due to Signature Science, LLC for use of the Omni Spray ionization source.

\section{References}

1. Venter, A.; Nefliu, M.; Cooks, R. G. Ambient Desorption Ionization Mass Spectrometry. Trends Anal. Chem. 2008, 27, 284-290.

2. Takats, Z.; Wiseman, J. M.; Cooks, R. G. Ambient Mass Spectrometry Using Desorption Electrospray Ionization (DESI): Instrumentation, Mechanisms and Applications in Forensics, Chemistry, and Biology. J. Mass Spectrom. 2005, 40, 1261-1275.

3. D'Agostino, P. A.; Hancock, J. R.; Chenier, C. L.; Lepage, J. Liquid Chromatography Electrospray Tandem Mass Spectrometric and Desorption Electrospray Ionization Tandem Mass Spectrometric Analysis of Chemical Warfare Agents in Office Media Typically Collected During a Forensic Investigation. J. Chromatogr. A 2006, 1110, 86-94.

4. Ifa, D. R.; Gumaelius, L. M.; Eberlin, L. S.; Manicke, N. E.; Cooks, R. G. Forensic Analysis of Inks by Imaging Desorption Electrospray Ionization (DESI) Mass Spectrometry. Analyst 2007, 132, 461-467.

5. Nyadong, L.; Green, M.D.; De Jesus, V. R.; Newton, P. N.; Fernandez, F. M. Reactive Desorption Electrospray Ionization Linear Ion Trap Mass Spectrometry of Latest-Generation Counterfeit Antimalarials via Noncovalent Complex Formation. Anal. Chem. 2007, 79, 2150-2157.

6. Cotte-Rodriguez, I.; Takats, Z.; Talaty, N.; Chen, H.; Cooks, R. G. Desorption Electrospray Ionization of Explosives on Surfaces: Sensitivity and Selectivity Enhancement by Reactive Desorption Electrospray Ionization. Anal. Chem. 2005, 77, 6755-6764.

7. Takats, Z.; Cotte-Rodriguez, I.; Talaty, N.; Chen, H.; Cooks, R. G. Direct, Trace Level Detection of Explosives on Ambient Surfaces by Desorption Electrospray Ionization Mass Spectrometry. Chem. Commun. 2005, 19501952.

8. Justes, D. R.; Talaty, N.; Cotte-Rodriguez, I.; Cooks, R. G. Detection of Explosives on Skin Using Ambient Ionization Mass Spectrometry. Chem. Commun. 2007, 21, 2142-2144.

9. Cotte-Rodriguez, I.; Hernandez-Soto, H.; Chen, H.; Cooks, R. G. In Situ Trace Detection of Peroxide Explosives by Desorption Electrospray Ionization and Desorption Atmospheric Pressure Chemical Ionization. Anal. Chem. 2008, 80, 1512-1519.

10. Cotte-Rodriguez, I.; Cooks, R. G. Non-Proximate Detection of Explosives and Chemical Warfare Agent Simulants by Desorption Electrospray Ionization Mass Spectrometry. Chem. Commun. 2006, 28, 2968pray
2970 .

11. D'Agostino, P. A.; Chenier, C. L.; Hancock, J. R.; Lepage, J. Desorption Electrospray Ionization Mass Spectrometric Analysis of Chemical Warfare Agents from Solid-Phase Microextraction Fibers. Rapid Commun. Mass Spectrom. 2007, 21, 543-549.

12. Song, Y.; Cooks, R. G. Reactive Desorption Electrospray Ionization for Selective Detection of the Hydrolysis Products of Phosphonate Esters. J. Mass Spectrom. 2007, 42, 1086-1092.

13. Cooks, R. G.; Ouyang, Z.; Takats, Z.; Wiseman, J. M. Ambient Mass Spectrometry. Science 2006, 311, 1566-1569.

14. Ifa, D. R.; Wiseman, J. M.; Qingyu, S.; Cooks, R. G. Development of Capabilities for Imaging Mass Spectrometry Under Ambient Conditions with Desorption Electrospray Ionization (DESI). Int. J. Mass Spectrom. 2007, 259, 8-15.
15. Wiseman, J. M.; Ifa, D. R.; Venter, A.; Cooks, R. G. Ambient Molecular Imaging by Desorption Electrospray Ionization Mass Spectrometry. Nat. Protocols 2008, 3, 517-524.

16. Chen, H; Talaty, N. N.; Takats, Z.; Cooks, R. G. Desorption Electrospray Ionization Mass Spectrometry for High Throughput Analysis of Pharmaceutical Samples in the Ambient Environment. Anal. Chem. 2005, 77, 6915-6927.

17. Van Berkel, G. J.; Ford, M. J.; Deibel, M. A. Thin-Layer Chromatography and Mass Spectrometry Coupled Using Desorption Electrospray Ionization. Anal. Chem. 2005, 77, 1207-1215.

18. Weston, D. J.; Bateman, R.; Wilson, I. D.; Wood, T. R.; Creaser, C. S Direct Analysis of Pharmaceutical Drug Formulations Using Ion Mobility Spectrometry/Quadrupole-Time-of-Flight Mass Spectrometry Combined with Desorption Electrospray Ionization. Anal. Chem. 2005, 77, 7752-7580.

19. Williams, J. P.; Scrivens, J. H. Rapid Accurate Mass Desorption Electrospray Ionization Tandem Mass Spectrometry of Pharmaceutical Samples. Rapid Commun. Mass Spectrom. 2005, 19, 3643-3650.

20. Hu, Q.; Talaty, N.; Noll, R. J.; Cooks, R. G. Desorption Electrospray Ionization Using an Orbitrap Mass Spectrometer: Exact Mass Measurements on Drugs and Peptides. Rapid Commun. Mass Spectrom. 2006, 20 3403-3408.

21. Williams, J. P.; Patel, V. J.; Holland, R.; Scrivens, J. H. The Use of Recently Described Ionization Techniques for the Rapid Analysis of Some Common Drugs and Samples of Biological Origin. Rapid Commun. Mass Spectrom. 2006, 20, 1447-1456.

22. Nyadong, L.; Green, M. D.; De Jesus, V. R.; Newton, P. N.; Fernandez, F. M. Reactive Desorption Electrospray Ionization Linear Ion Trap Mass Spectrometry of Latest-Generation Counterfeit Antimalarials via Noncovalent Complex Formation. Anal. Chem. 2007, 79, 2150-2157.

23. Ricci, C.; Nyadong, L.; Fernandez, F. M.; Newton, P. N.; Kazarian, S. G. Combined Fourier-Transform Infrared Imaging and Desorption Electrospray-Ionization Linear Ion-Trap Mass Spectrometry for Analysis of Counterfeit Antimalarial Tablets. Anal. Bioanal. Chem. 2007, 387, $551-559$.

24. Ifa, D. R.; Manicke, N. E.; Rusine, A. L.; Cooks, R. G. Quantitative Analysis of Small Molecules by Desorption Electrospray Ionization Mass Spectrometry from Polytetrafluoroethylene Surfaces. Rapid Commun. Mass Spectrom. 2008, 22, 503-510.

25. Talaty, N.; Takats, Z.; Cooks, R. G. Rapid In Situ Detection of Alkaloids in Plant Tissue Under Ambient Conditions using Desorption Electrospray Ionization. Analyst 2005, 130, 1624-1633.

26. Jackson, A. T.; Williams, J. P.; Scrivens, J. H. Desorption Electrospray Ionization Mass Spectrometry and Tandem Mass Spectrometry of Low Molecular Weight Synthetic Polymers. Rapid Commun. Mass Spectrom. 2006, 20, 2717-2727.

27. Williams, J. P.; Hilton, G. R.; Thalassinos, K.; Jackson, A. T.; Scrivens, J. H. The Rapid Characterization of Poly(Ethylene Glycol) Oligomers Using Desorption Electrospray Ionization Tandem Mass Spectrometry Combined with Novel Product Ion Peak Assignment Software. Rapid Commun. Mass Spectrom. 2007, 21, 1693-1704.

28. Chen, H.; Zhengzheng, P.; Talaty, N.; Raftery, D.; Cooks, R. G. Combining Desorption Electrospray Ionization Mass Spectrometry and Nuclear Magnetic Resonance for Differential Metabolomics without Sample Preparation. Rapid Commun. Mass Spectrom. 2006, 20, 1577-1584.

29. Kauppila, T. J.; Wiseman, J. M.; Ketola, R. A.; Kotiaho, T.; Cooks, R. G.; Kostiainen, R. Desorption Electrospray Ionization Mass Spectrometry for the Analysis of Pharmaceuticals and Metabolites. Rapid Commun. Mass Spectrom. 2006, 20, 387-392.

30. Pan, Z.; Gu, H.; Talaty, N.; Chen, H.; Shanaiah, N.; Hainline, B. E. Cooks, R. G.; Raftery, D. Principal Component Analysis of Urine Metabolites Detected by NMR and DESI-MS in Patients with Inborn Errors of Metabolism. Anal. Bioanal. Chem. 2007, 387, 539-549.

31. Jackson, A. U.; Werner, S. R.; Talatly, N.; Song, Y.; Campbell, K.; Cooks, R. G.; Morgan, J. A. Targeted Metabolomic Analysis of Escherichia Coli by Desorption Electrospray Ionization and Extractive Electrospray Ionization Mass Spectrometry. Anal. Biochem. 2008, 375, 272-281.

32. Bereman, M. S.; Nyadong, L.; Fernandez, F. M.; Muddiman, D. C. Direct High-Resolution Peptide and Protein Analysis by Desorption Electrospray Ionization Fourier Transform Ion Cyclotron Resonance Mass Spectrometry. Rapid Commun. Mass Spectrom. 2006, 20, 3409-3411.

33. Shin, Y-S.; Drolet, B.; Mayer, R.; Dolence, K.; Basile, F. Desorption Electrospray Ionization-Mass Spectrometry of Proteins. Anal. Chem. 2007, 79, 3514-3518.

34. Bereman, M. S.; Williams, T. I.; Muddiman, D. C. Carbohydrate Analysis by Desorption Electrospray Ionization Fourier Transform Ion Cyclotron Resonance Mass Spectrometry. Anal. Chem. 2007, 79, 88128815.

35. Venter, A.; Cooks, R. G. Desorption Electrospray Ionization in a Small Pressure-Tight Enclosure. Anal. Chem. 2007, 79, 6398-6403.

36. Chipuk, J. E.; Brodbelt, J. S. Transmission Mode Desorption Electrospray Ionization. J. Am. Soc. Mass Spectrom. 2008, 19, 1612.

37. Kauppila, T. J.; Talaty, N.; Salo, P. K.; Kotiaho, T.; Kostiainen, R.; Cooks, R. G. New Surfaces for Desorption Electrospray Ionization Mass Spectrometry: Porous Silicon and Ultra-Thin Layer Chromatography Plates. Rapid Commun. Mass Spectrom. 2006, 20, 2143-2150.

38. Volný, M.; Venter, A.; Smith, S. A.; Pazzi, M.; Cooks, R. G. Surface Effects and Electrochemical Cell Capacitance in Desorption Electrospray Ionization. Analyst 2008, 133, 525-531. 
39. Sen, A. K.; Nayak, R.; Darabi, J.; Knapp, D. R. Use of Nanoporous Alumina Surface for Desorption Electrospray Ionization Mass Spectrometry in Proteomic Analysis. Biomed. Microdevices 2008, 10, 531-538.

40. Costa, A. B.; Cooks, R. G. Simulation of Atmospheric Transport and Droplet-Thin Film Collisions in Desorption Electrospray Ionization. Chem. Commun. 2007, 38, 3915-3917.

41. Electrospray Ionization Mass Spectrometry: Fundamentals, Instrumentation, and Applications, Cole, R. B., Ed.; Wiley: New York, 1997.

42. Cech, N. B.; Enke, C. G. Practical Implications of Some Recent Studies in Electrospray Ionization Fundamentals. Mass Spectrom. Rev. 2001, 20, 362-387.

43. Page, J. S.; Kelly, R. T.; Tang, K.; Smith, R. D. Ionization and Transmission Efficiency in an Electrospray Ionization-Mass Spectrometry Interface. J. Am. Soc. Mass Spectrom. 2007, 18, 1582-1590.

44. Pasilis, S. P.; Kertesz, V.; Van Berkel, G. J. Surface Scanning Analysis of Planar Arrays of Analytes with Desorption Electrospray IonizationMass Spectrometry. Anal. Chem. 2007, 79, 5956-5962.

45. Shiea, J.; Huang, M.; Hsu, H.; Lee, C.; Yuan, C.; Beech, I.; Sunner, J. Electrospray-Assisted Laser Desorption/Ionization Mass Spectrometry for Direct Ambient Analysis of Solids. Rapid Commun. Mass Spectrom. 2005, 19, 3701-3704.
46. Peng, I. X.; Shiea, J.; Loo, R. R. O.; Loo, J. A. Electrospray-Assisted Laser Desorption/Ionization and Tandem Mass Spectrometry of Peptides and Proteins. Rapid Commun. Mass Spectrom. 2007, 21, 2541-2546.

47. Shiea, J.; Yuan, C.-H.; Huang, M.-Z.; Cheng, S.-C.; Ma, Y.-L.; Tseng, W.-L.; Chang, H.-C.; Hung, W.-C. Detection of Native Protein Ions in Aqueous Solution under Ambient Conditions by Electrospray Laser Desorption/Ionization Mass Spectrometry. Anal. Chem. 2008, 80, 48454852.

48. Sampson, J. S.; Hawkridge, A. M.; Muddiman, D. C. Generation and Detection of Multiply-Charged Peptides and Proteins by MatrixAssisted Laser Desorption Electrospray Ionization (MALDESI) Fourier Transform Ion Cyclotron Resonance Mass Spectrometry. J. Am. Soc. Mass Spectrom. 2006, 17, 1712-1716.

49. Sampson, J. S.; Hawkridge, A. M.; Muddiman, D. C. Direct Characterization of Intact Polypeptides by Matrix-Assisted Laser Desorption Electrospray Ionization Quadrupole Fourier Transform Ion Cyclotron Resonance Mass Spectrometry. Rapid Commun. Mass Spectrom. 2007, 21, $1150-1154$.

50. Sampson, J. S.; Hawkridge, A. M.; Muddiman, D. C. Development and Characterization of an Ionization Technique for Analysis of Biological Macromolecules: Liquid Matrix-Assisted Laser Desorption Electrospray Ionization. Anal. Chem. 2008, 80, 6773-6778. 